\title{
El significado del diagnóstico en la trayectoria del enfermo reumático: De la incertidumbre a la disrupción biográfica
}

\author{
The meaning of the diagnosis in the illness trajectory \\ of a person with rheumatic disease: From the \\ uncertainty to biographical disruption
}

${ }^{1}$ Doctora en Antropología Social y Cultural. Profesora-Investigadora Titular, Centro de Investigaciones $y$ Estudios Superiores en Antropología Social (CIESAS), México. $\square$
RESUMEN El interés de este artículo es analizar el significado del diagnóstico médico en la biografía de los sujetos que padecen alguna enfermedad reumática, a través de un trabajo etnográfico basado en entrevistas en profundidad realizadas a un grupo de quince informantes de la Ciudad de Barcelona afectados por distintos padecimientos reumáticos. Los relatos de los entrevistados permiten abordar cómo los síntomas, disfunciones o limitaciones, experimentadas hasta el momento en que se emite un diagnóstico certero, finalmente pueden ser interpretados a la luz de un modelo que explica esta condición, se reconoce cuál es la enfermedad, se resignifica la sintomatología y se establecen determinadas estrategias de atención y afrontamiento. En este sentido, el diagnóstico médico definitivo logra descifrar el enigma de la condición crónica que aqueja al enfermo y constituye así un punto de inflexión en la trayectoria de atención, que es reconocido como una disrupción biográfica que resignifica el pasado y futuro del sujeto. No obstante, con frecuencia los afectados recorren un complejo itinerario, a veces errático e incierto, para llegar al diagnóstico definitivo, por lo que este momento se identifica retrospectivamente como un hito en la experiencia del padecimiento.

PALABRAS CLAVES Diagnóstico Clínico; Enfermedades Reumáticas; Enfermedad Crónica; Personas con Discapacidad.

\begin{abstract}
This article seeks to analyze the meaning of the medical diagnosis in the biographies of people who suffer from a rheumatic disease. Based in the ethnographic method, in-depth interviews were carried out among fifteen informants affected by different rheumatic conditions from the City of Barcelona. These interviews make it possible to see how the symptoms, dysfunctions or limitations experienced up until the definitive diagnosis can be reinterpreted in the presence of a model that explains the condition and gives it a name, attributing new meaning to the symptoms and establishing certain care and coping strategies. After a period of uncertainty, this definitive medical diagnosis allows for an enigmatic chronic condition from which the person is suffering to be deciphered, representing a turning point in the care trajectory that is understood as a biographical disruption redefining the past and future of the subject. However, it is often necessary for those affected to undergo a complex, erratic and uncertain itinerary to reach the final diagnosis, which is then identified retrospectively as a milestone in the illness experience.
\end{abstract}

KEY WORDS Clinical Diagnosis; Rheumatic Diseases; Chronic Disease; Disabled Persons. 


\section{INTRODUCCIÓN}

El trabajo se enmarca en una investigación realizada en la ciudad de Barcelona, que tiene como objetivo global el análisis de la cronicidad, definida como el proceso sociocultural y subjetivo que se construye a través de la experiencia particular de aqueIlos sujetos que se encuentran en la situación de convivir cotidianamente, de manera prolongada y/o permanente con un daño, enfermedad, disfunción o discapacidad definida como "crónica". La investigación se organizó en torno a varios aspectos que se hallan estrechamente interrelacionados, pero que para fines analíticos y expositivos, se estructuran en cuatro ejes principales: la experiencia del padecimiento en el marco de la biografía del sujeto; la trayectoria del padecimiento centrada en los itinerarios diagnósticos y terapéuticos; las principales consecuencias físicas, sociales, psicológicas y económicas derivadas de la enfermedad; $y$, finalmente, la corporalidad y su impacto en la subjetividad del enfermo ${ }^{(1)}$.

Se considera que la aparición de cualquier proceso enfermante puede alterar en mayor o menor grado la vida cotidiana del sujeto, dependiendo de la limitación funcional o social derivada, de su gravedad, de su duración, o de las estrategias necesarias para su atención, entre otros aspectos. Sin embargo, cuando una condición se constituye en una constante en la vida del sujeto, y a través de un diagnóstico profesional biomédico, se reconoce/etiqueta a ese sujeto como un "enfermo crónico", dicho reconocimiento constituye un hecho particularmente significativo en la biografía del afectado, tanto por el hecho de que la curación es altamente improbable, como porque su duración y evolución pueden ser inciertas. En algunos casos, la condición médica puede ser controlable, más o menos estable, o bien presentar periodos de crisis y remisión, mientras que, en otros casos, puede estar marcado por un proceso degenerativo e incluso de consecuencias letales. Sea de índole leve o grave, lo que tiene en común es su carácter vitalicio y la necesidad de que los sujetos aprendan a vivir con (y a pesar de) su condición crónica.

El enfermo crónico experimenta y aprende a reconocer un conjunto de huellas que no son únicamente físicas, sino emocionales y sociales, que el padecer va inscribiendo en su cuerpo, en su identidad, en su biografía. Las formas en que cada sujeto vive, enfrenta e interpreta su padecimiento, no solo moldean su estilo de vida o inciden en sus relaciones sociales, sino que van reconfigurando la propia subjetividad. En este sentido, las huellas del padecimiento se concretan -a veces de manera contundente- $y$ nos permiten analizar de qué manera la cronicidad moldea o estructura al sujeto, se inscribe en el cuerpo, en un sentido literal y metafórico y se incorpora en el sujeto (embodied) $^{(2)}$.

En estos padecimientos, la terapéutica médica se orienta hacia formas de mantenimiento, control y limitación del daño y a establecer pautas de prevención secundaria y/o cuidados paliativos en un horizonte terapéutico cuyos límites extremos se ubican entre el control y la muerte. El padecimiento crónico define un proceso en el que el sujeto deberá aprender a convivir con él, generando estrategias de afrontamiento de tipo individual o grupal.

El objetivo de este trabajo es describir y analizar cuál fue el impacto que tuvo el diagnóstico médico en la experiencia del padecimiento. A través de la mirada retrospectiva de los propios afectados se busca reconstruir cómo se recibió el diagnóstico, cómo se resignificó el conjunto de síntomas, disfunciones o limitaciones experimentadas hasta ese momento y que a partir de entonces pudieron ser reconocidas con un nombre que facilitó su comprensión y abrió un nuevo camino a seguir: el momento diagnóstico se registra como un punto de inflexión en el itinerario diagnóstico de las enfermedades reumáticas y deja una huella en la vida de nuestros entrevistados, un punto de Ilegada $y$, al mismo tiempo, un inicio en su experiencia del padecer. 


\section{CONTEXTO DE LA INVESTIGACIÓN Y PERFILES DE LOS ENTREVISTADOS}

La información etnográfica que se presenta en este trabajo procede de una investigación sobre procesos de cronicidad que se desarrolló en la ciudad de Barcelona en varios periodos de trabajo de campo entre los años 2005 y 2012, en la que trabajé con distintos grupos de enfermos crónicos o en situación de dependencia y discapacidad. Me centré en analizar las significaciones y consecuencias que la condición crónica ha tenido para la vida de estas personas y para quienes las rodean.

Este caso se basa en entrevistas en profundidad realizadas a un grupo de personas afectadas por distintos padecimientos reumáticos, para lo cual entré en contacto con una asociación de enfermos, mediante la cual contacté a quince informantes, socios de la asociación, que participaban, en mayor o menor grado, en sus actividades. Adicionalmente, entrevisté a diversos profesionales sanitarios que habían trabajado con esta asociación. A la gran mayoría de ellos se los entrevistó en dos o tres sesiones y el tiempo promedio dedicado a cada uno fue de seis horas, aproximadamente. Todos aceptaron participar en la investigación, a través de un consentimiento informado y con el compromiso de mantener el anonimato y la confidencialidad de la información.

Nuestros informantes tenían un perfil relativamente heterogéneo en cuanto al tipo y duración del padecimiento reumático, aunque compartían algunas características socioeconómicas. Se seleccionaron adultos jóvenes o maduros, de ambos sexos, que hayan sido afectados hacía más de cinco años por algún tipo de padecimiento reumático y que estuviesen dispuestos a participar en la investigación. Se entrevistaron diez mujeres y cinco hombres, con un rango de edad entre los 30 y los 70 años, aunque la mitad de ellos se encontraban cerca de los 50 años. La edad promedio del grupo era de 48 años.

De los quince informantes, nueve estaban casados, dos viudas, dos divorciadas y dos solteras. Cuatro de ellas, las más jóvenes, eran menores de 40 años, no tenían hijos y habían optado por la no maternidad, en una decisión principalmente derivada de su padecimiento. Trece de los informantes vivían con su grupo familiar y únicamente dos de ellos vivían solos.

En términos socioeconómicos, en general, pertenecían a sectores medios, la mayoría tenía un nivel de educación superior y se habían desempeñado como profesionales o bien, habían realizado carreras técnicas. Sin embargo, hacía tiempo que se habían retirado de las labores productivas, ya que quienes habían tenido una vida laboral activa, se habían jubilado por la enfermedad. Todos tenían un reconocimiento biomédico y jurídico que les acreditaba su limitación parcial o total para desempeñar una vida laboral y a consecuencia de su enfermedad, se encontraban pensionados. Los varones expresaban mayores preocupaciones económicas derivadas de la enfermedad, pues habían pasado de ser sostén único de la familia, a ser proveedores secundarios, aportando solo su pensión al gasto familiar, mientras que sus esposas se habían tenido que incorporar al mercado laboral presionadas por esta situación.

Los informantes padecían diversos tipos de enfermedades reumáticas, a veces asociadas con otros padecimientos crónicos. Cuatro padecían artritis reumatoide, dos esclerodermia, tres espondilitis anquilosante, tres fibromialgia, dos lupus y, una, artrosis. En los casos de comorbilidad, se había diagnosticado depresión, ceguera, diabetes, trastorno obsesivo compulsivo y accidente vascular cerebral.

Tanto los más jóvenes como los adultos mayores tenían una larga experiencia del padecimiento, la tercera parte de ellos llevaba treinta años sufriéndola, casi la mitad tenía entre quince y veinticinco años, tres la habían sufrido cerca de diez años y solo una llevaba cinco años. La edad del sujeto y la duración del padecimiento marcaban sustantivamente sus biografías, pues representaba que la enfermedad irrumpió antes de los 20 años en seis casos y, en los nueve restantes, antes de los 45 años. Todos fueron afectados a una edad joven, en plena etapa productiva 
y reproductiva, lo que alteró drásticamente su vida laboral, o modificó sus proyectos de maternidad y sus relaciones de pareja, como sucedió a las cuatro mujeres más jóvenes que entrevistamos.

Un aspecto sobresaliente que necesita ser explicitado respecto del perfil de los entrevistados es que todos tenían cierta experiencia de participación en grupos de ayuda mutua y en asociaciones de enfermos, incluso algunos de ellos eran destacados activistas, por lo que habían tenido tiempo y espacio para reflexionar colectivamente sobre los problemas derivados de su padecer. La pertenencia a una asociación de afectados, favorecía su disposición a hablar con mayor soltura sobre la experiencia de su padecimiento, habían tenido ocasión de presentarse a sí mismos como afectados por procesos reumáticos ante la mirada de los otros, de contar su historia, no una sino varias veces. Esto hace que el relato se construya con los alcances y limitaciones de la narrativa biográfica que menciona Bourdieu $^{(3)}$ cuando señala que el narrador reconstruye el relato como una ilusión biográfica, destaca aspectos relevantes que son retrospectivamente resaltados, en tanto que otros aspectos son minimizados, olvidados o negados consciente o inconscientemente por el narrador, sobre la base de sus supuestos acerca de las expectativas creadas o imaginadas de quien escucha. Desde mi punto de vista, desarrollaron una reconstrucción de su biografía quizás más reflexiva, en la que el padecimiento adquiere un papel relevante en su identidad, no solo por la experiencia en sí misma, sino porque una de las estrategias encontradas para hacerle frente era, justamente, la participación activa en el grupo de autoayuda.

\section{EL ITINERARIO DIAGNÓSTICO: DE LA INCERTIDUMBRE A LA DISRUPCIÓN BIOGRÁFICA}

En nuestro trabajo nos referimos al "itinerario diagnóstico" como aquella fase de la trayectoria de atención en la que se identifican los primeros síntomas, se busca la explicación causal asociada a determinada entidad nosológica, se consultan uno o varios profesionales, que pueden $-\mathrm{O}$ no- instrumentar pruebas diagnósticas y/o terapéuticas a fin de establecer interpretaciones profesionales o diagnósticos tentativos o provisionales respecto de la condición de enfermo, hasta llegar al establecimiento del diagnóstico correcto o definitivo. Si bien se identifica como una etapa inicial en la trayectoria del padecimiento, su duración suele ser variable en el tiempo, ya que puede prolongarse a través de varios diagnósticos provisionales, confusos o equivocados y de tratamientos destinados a paliar determinados síntomas, pero no siempre idóneos.

El itinerario diagnóstico así entendido puede ser corto y certero, pero -como fue el caso en casi todos nuestros informantestambién puede llegar a constituirse en un difícil recorrido marcado por la incertidumbre de no saber qué es lo que sucede, pasar por varios especialistas de distinto orden, cada cual observando solo un aspecto parcial del padecer sin llegar a precisar la entidad nosológica con la que se enfrentaban, sin atar cabos para emitir un diagnóstico correcto. Este tránsito ha sido descrito por nuestros informantes como "un laberinto en la oscuridad", "caminar sin saber muy bien cuál es el camino por el que se está yendo", "dando palos de ciego", y a los malestares físicos se suman la incertidumbre, la ansiedad o la preocupación.

En este difícil recorrido, el establecimiento de un diagnóstico preciso puede representar un alivio para el enfermo, en tanto da fin a este desasosiego y le permite nombrar-etiquetar- el conjunto de síntomas que se manifiestan, hasta entonces, de manera difusa y desconcertante. En ciertas fases de la enfermedad, poder establecer un diagnóstico acertado puede significar, finalmente, obtener una explicación consistente de los síntomas, que brinde al sujeto un encuadre que le permita comprender los procesos corporales por los cuales está atravesando y se encamine con mayor certeza en la búsqueda de una atención orientada hacia objetivos 
más definidos, quizás con la esperanza de que por esta vía encuentre una posible solución a sus problemas. No obstante, este componente de certeza puede significar, paralelamente, una grave noticia, pues saber que el padecimiento es crónico, no curable o de pronóstico severo, en ocasiones es interpretado como si fuese una "sentencia" que condenará al individuo a vivir, sentir y construir su mundo en relación con este padecer.

Su principal importancia radica en que, al llegar al punto de recibir un diagnóstico definitivo, se identifica un punto de inflexión en la manera en que el sujeto se aproxima a la naturaleza de su enfermedad. Puede, por fin, nombrarlo. En algunos casos, no se toma conciencia plena de su significado pero, en otros, se reconoce el etiquetamiento como crónico o "vitalicio", y puede proceder a actuar en consecuencia, modificando en parte su estilo de vida, o elaborando cierta reflexión sobre su condición presente o futura, o replanteándose incluso algunos aspectos de su identidad. En este sentido, el diagnóstico de la enfermedad permite identificarla, certificarla y normalizarla; el diagnóstico constituye un hito, un punto de partida de una situación personal que se reconoce como permanente y vitalicia ${ }^{(4)}$. El diagnóstico opera, en la mayoría de los casos, como un "antes y un después" en la vida del sujeto.

El anuncio del diagnóstico puede resultar crucial en la biografía de los individuos afectados, ya que no solo es un diagnóstico médico, sino que incide en la reconfiguración de la propia identidad de la persona ${ }^{(5,6)}$. EI momento en que conoce el diagnóstico y su impacto varía mucho de acuerdo con las historias personales. Este acontecimiento puede significar, en algunos casos, una continuidad con el pasado, un paso inexorable hacia un futuro incierto, o un resultado esperable $y$, hasta cierto punto, predecible en concordancia con el estilo de vida que se venía llevando o, por el contrario, puede representar un momento de ruptura en la historia personal del sujeto.

En este sentido, considero relevante plantear una de las perspectivas de análisis que han marcado el debate teórico en torno a la cronicidad, planteada por Michael Bury ${ }^{(7)}$ en su estudio clásico de un grupo de pacientes con artritis reumatoide. Propone que las enfermedades crónicas son un tipo de experiencia en que la estructura de la vida cotidiana y las formas de conocimiento que la sostienen se trastornan, perturban, alteran en su flujo cotidiano. Bury sugiere analizar las enfermedades crónicas a través de la categoría de disrupción biográfica (biographical disruption), que más que una ruptura propiamente dicha, significaría la alteración del flujo normal de las cosas, la incapacidad de continuar de manera natural las actividades.

La etiqueta diagnóstica ha sido analizada en términos de una disrupción en la biografía de quien lo sufre, un antes y un después en su historia personal, que lo llevan a plantearse preguntas del tipo ¿Qué me está pasando?, ¿por qué sucede?, ¿qué me depara el futuro?, ¿qué debo hacer? Interrogantes que simbolizan la incertidumbre, el dolor y el sufrimiento que pueden ir desde la negación, el escape de la realidad, la autoculpabilización, o el rechazo a asumir la nueva condición.

Bury identifica dos etapas en este proceso. La primera se refiere al inicio del problema y el reconocimiento de la nueva condición; requiere explicar lo que pasa y se observa una disrupción de los aspectos o conductas que hasta ese momento se han dado por sentadas desde el sentido común. Esta etapa involucra una atención consciente de los estadios corporales, así como las decisiones de buscar ayuda; se detecta un cambio biográfico de una trayectoria percibida como normal o predecible, hacia una fundamentalmente atípica, incierta o perjudicial ${ }^{(7)}$. Ya que los primeros daños o síntomas se identifican como una molestia, a veces se evalúan como si fuesen el producto de alguna actividad atípica, pero no son interpretados como una posible señal de peligro. El inicio de las enfermedades es incierto, imperceptible, silencioso y muchas veces asintomático. Es probable que en esta etapa no se involucre a sus redes cercanas, sino hasta un estadio avanzado de la enfermedad. En ocasiones -señala Bury ${ }^{(7,8)}$ - los pacientes ocultan sus síntomas o disfrazan sus efectos por largos 
períodos. La búsqueda de ayuda profesional especializada y el diagnóstico marcan el final de esta primera etapa. Se genera una sensación de alivio por poder denominar el nuevo estado, aunque al mismo tiempo hay una reacción de incredulidad y ansiedad.

La segunda etapa implicaría una serie de disrupciones o rupturas más profundas en los sistemas explicativos normalmente usados por la gente, que propician un replanteamiento fundamental de la biografía de la persona y movilizan los recursos ante la situación alterada. Esta etapa aparece marcada por la emergencia de la discapacidad, el problema de la incertidumbre sobre el impacto y el curso de la enfermedad así como cuál debe ser la conducta apropiada respecto a sus efectos.

A partir de aquí se da una búsqueda de legitimidad y tolerancia de los otros, lo que implica una re-evaluación de la relación entre la enfermedad que ahora ya es manifiesta y su identidad propia. El diagnóstico provee de una información firme para relacionarse con el nuevo estado y explicarlo a los otros, pero la eficacia del tratamiento (a veces por ensayo-error) y el conocimiento sobre las causas son limitadas, por lo que aparecen sensaciones encontradas de miedo, alivio, ansiedad. Se hace necesario un puente que vincule esta información, el conocimiento formal de la enfermedad con la biografía total de la persona, que le dé respuestas a los diversos porqués que se le plantean y que, a través de una revisión biográfica, pueda encontrar una causalidad subjetivamente significativa ${ }^{(9)}$.

Junto a esta disrupción de significados y estructuras profundas, también se verifica una alteración del estilo de vida, un cambio en la vida cotidiana, sus actividades, su gestión del padecer, tanto en términos materiales como organizacionales, una reorganización práctica en sus relaciones y en la red de apoyo social ${ }^{(10)}$. La disrupción de los vínculos de amistad o comunitarios surgen no solo por las limitaciones funcionales, sino también debido al estigma o al sentimiento de vergüenza u ocultamiento que la cronicidad y la discapacidad crean, produciendo un abandono de ciertas esferas y núcleos de socialización y en ocasiones un creciente aislamiento social ${ }^{(11)}$.

En este sentido, es importante tomar en cuenta que existen diversos aspectos que van moldeando la intensidad e impacto de las disrupciones en la vida del sujeto, tales como las representaciones sociales que se tengan sobre la enfermedad, -sean previas a su experiencia o derivadas de esta-, la ausencia o presencia de una red de apoyo social, principalmente dentro del grupo familiar, los escenarios de interacción en los que desarrollan sus actividades, la posibilidad de legitimar cambios en la conducta, así como su capacidad de resiliencia frente a la discapacidad y el estigma ${ }^{(12,13)}$.

Las consecuencias físicas, psicológicas, sociales o económicas que conllevan las enfermedades crónicas alteran no solo la vida del enfermo, sino que introducen cambios en su entorno y pueden alterar la dinámica del grupo familiar tanto en un sentido positivo, al activar un sistema de cuidados y apoyos solidarios al enfermo, o bien de manera negativa, cuando operan como catalizador de los conflictos preexistentes, que se agudizan con la nueva condición. En cualquier caso, las reglas usuales de la reciprocidad y el apoyo mutuo se ven alteradas en función de la dependencia creciente del enfermo ${ }^{(6)}$.

Este enfoque sobre la disrupción biográfica ha recibido diversas críticas ${ }^{(14,15,16,17)}$, aunque también ha recibido el impulso de múltiples investigaciones que comparten esta perspectiva de análisis ${ }^{(8,18)}$ y de alguna manera se ha convertido en una referencia obligada dentro de los estudios sobre cronicidad ${ }^{(19,20,21,22,23,24,25)}$.

Desde nuestra experiencia de investigación con este grupo de enfermos reumáticos, la disrupción biográfica ha sido referida de manera consistente en mayor o menor intensidad por el conjunto de nuestros entrevistados, todos reconocieron la manera en que la enfermedad trastocó sus biografías, sus actividades, relaciones y proyectos. Nos hablaban de cómo habían aprendido a convivir con ella, asumiendo las limitaciones, generando estrategias de afrontamiento, adaptándose a 
un nuevo estilo de vida, reconfigurando su propia identidad. Mencionaron un permanente proceso de aprendizaje para reconocer la evolución de ciertos síntomas, monitorear el cuerpo y reconocer las crecientes limitaciones producidas e identificar aquellos riesgos reales o imaginarios que debían evitar.

En este sentido es que recuperamos el concepto de incorporación (embodiment) de Csordas ${ }^{(2)}$, para referirnos a aquellas formas de apropiación del padecimiento que se expresan en el cuerpo y a través del cuerpo; el padecimiento crónico se incorpora a la identidad del afectado. Gran parte de su vida y de quienes lo rodean gira en torno al eje de la enfermedad, su mundo se va redefiniendo a partir de lo que puede hacer (limitaciones) y de lo que debe evitar (riesgos) y cómo controlar ambas dimensiones dentro del marco de unas estrategias de afrontamiento en un proceso permanente de adaptación y monitoreo corporal; una parte de su rutina cotidiana pasa por las actividades de autoatención y atención profesional a la enfermedad, tanto en términos de terapéutica y rehabilitación, como de la gestión y obtención de los prestaciones sociales que provee el Estado. Estas actividades de gestión social implican transitar por un complejo entramado de instituciones, legislaciones y procedimientos administrativos existentes a fin de tramitar las pensiones por discapacidad, las bajas laborales, los certificados de disminución, que para los entrevistados o sus familiares, significaban un recorrido prolongado y bastante dificultoso.

\section{Una mirada retrospectiva al momento del diagnóstico}

En este apartado se desarrolla la reconstrucción retrospectiva que nuestros entrevistados hacen de su propio itinerario diagnóstico, un proceso más o menos largo, que ha quedado plasmado con cierta nitidez en la memoria de los entrevistados. Algunos de los primeros síntomas identificados fueron la aparición de dolor o inflamación recurrentes en algunas articulaciones $\mathrm{u}$ otras zonas del cuerpo, que se hicieron cada vez más frecuentes e intensas. Su explicación causal (tanto por el enfermo como por los médicos consultados) fue asociada a determinada actividad realizada, a cierta postura inadecuada, o simplemente se la consideró de naturaleza "inexplicable".

Se mantuvo esta sintomatología durante largo tiempo, en el que no se sabía a ciencia cierta cuál era la enfermedad que lo aquejaba, se emitieron diagnósticos equívocos o difusos y se administraron tratamientos -básicamente antiinflamatorios o analgésicosque eran simplemente paliativos:

Empecé a los 17 años con unos dolores muy fuertes, de un día para otro me levanté con un dolor muy fuerte en un pie. Y a partir de ese momento, empecé en manos de médicos porque empecé a tener dolores en todo el cuerpo. Primero fue en los pies, luego en la rodilla, luego al otro día con las manos, luego al cabo de una semana la otra rodilla, al cabo de un mes el hombro. Como los dolores fueron primero en el pie me dijeron que era un esguince. Dije, es imposible porque yo no he hecho nada, y bueno era un esguince. Pero cuando ya fueron dolores en diferentes partes, primero las rodillas, y luego los hombros y dije que eso no podía ser, no era un esguince. Ese fue el médico de cabecera. Así empecé, con dolores intermitentes y era ir de médico tras médico, sea medicina natural, traumatólogos, médicos de cabecera, de todo tipo para saber qué era lo que tenía, íbamos a preguntar cualquier tipo de médicos porque no sabíamos para dónde tirar. Al final, al cabo de no sé cuánto tiempo, medio año, cinco meses, no lo sé, me diagnosticaron una "artritis". En ese momento, cuando me lo dijeron yo tenía 18 años. Fue al cabo de cinco meses, algo así, pero menos de un año, seguro. (Lety, 32 años. Artritis reumatoide juvenil)

La duración de la enfermedad es determinante en la manera en que se ha logrado incorporar en la identidad del enfermo. Como 
en el caso mencionado, casi la mitad de la vida de Lety, ha tenido que lidiar con las consecuencias de su enfermedad, bastante limitante, por cierto. Al recuperar la temporalidad del padecimiento en los entrevistados, el establecimiento del diagnóstico involucra una redefinición del pasado/presente y futuro de la enfermedad y también de él (ella) como sujeto, ya que aun cuando su identidad va más allá de la simple categoría de "enfermo crónico", puede constituirse en un núcleo muy significativo de su identidad:

La enfermedad se apareció a los diez años y bueno me tratan los médicos y yo no tengo conciencia de tener una enfermedad. Y bueno, pienso que es un mal que se me ha presentado y bueno me pasará y ya me curaré. Comencé con la rodilla inflamada y eso que impedía correr o jugar y ahí mis padres me llevaron al médico y me mandó un tratamiento, reposo y cede la inflamación, esto que repite hasta los 14 ó 15 años, y la cosa es seria, cada vez más severa, la inflamación es más resistente más acentuada y con 16 años me intervienen de la rodilla derecha. No, no me dicen nada (de diagnóstico). No saben qué es. Pensaban que era algún problema de la articulación y entonces lo que me diagnostican es que había una masa vinosa dentro de la articulación, o sea de color vino, gelatinosa. Me la extraen. Pero esto es de palabras, yo no tengo informes y me recetan radioterapia. No lo sé si pensaban que podía ser cáncer. Había algunos comentarios de que pudiera ser un tumor blanco, pero nunca supe que era eso, ni si era bueno o si era malo. Y luego resulta que no, no era del llamado cáncer de los huesos. Entonces me empiezan a las sesiones con radioterapia y desaparece los síntomas hasta los 19 años. (Pedro, 59 años. Espondilitis y ceguera)

Algunas líneas de análisis surgen de estos testimonios. El periodo de incertidumbre diagnóstica es típico de las enfermedades reumáticas, puesto que lleva cierto tiempo en emitir el diagnóstico, y porque el itinerario terapéutico muchas veces pasa por el médico de cabecera y cuando se acude al especialista, generalmente, se llega primero a través de la derivación al médico traumatólogo u ortopedista, el cual, en el mejor de los casos, deriva al paciente al reumatólogo quien, a partir de su especialidad, puede establecer un diagnóstico con mayor precisión.

Llama la atención que se repita la misma estructura en el itinerario diagnóstico: sintomatología difusa, diagnósticos inexactos y, al cabo de un tiempo, y otras tantas prescripciones terapéuticas (analgésicos, antiinflamatorios o corticoides), se realiza alguna prueba diagnóstica con cierto grado de especificidad (por ejemplo, identificación de factor reumatoide en sangre, estudios de radioimagen), que permita confirmar o descartar el diagnóstico presuntivo. También puede ser un indicador, la presencia de un brote muy agresivo, que permitió establecer un diagnóstico definitivo en un plazo relativamente más corto, como en el caso que se describe a continuación:

Todo fue hace ocho años, cuando tenía 29 años de edad, pues yo recuerdo que empecé a notar algo raro porque se me hinchaban los tobillos, tenía los tobillos hinchados y fue durante el verano, yo pensé que era de calor, pero como la hinchazón no se pasaba, pues en septiembre ya fui al médico porque además empezaba a tener dolor en las rodillas y dolores articulares. Y fui al médico como eran dolores articulares lo que yo sentía fui a trauma, y en trauma no me encontraron nada. Y bueno también empecé a hacer esguinces como con mucha facilidad. Si tú vas a trauma y hay dolor y no hay rotura es un esguince, pero no eran reales. $Y$ como en trauma no me encontraban nada, pasé a reuma. [...] Pasaron unos cuatro o cinco meses. Desde que comencé con estos dolores, empezaría por ahí de septiembre y el diagnóstico final lo tuve sobre febrero o así. En un lapso de seis meses comencé a tener 
muchos síntomas. Como si se hubiera disparado por así decirlo. Y el médico me dijo primero lupus, y en ese tiempo ya se me empezaron a manifestar todos los síntomas y ya empecé a tener la piel dura. Se me empezó a poner la piel dura y al poco tiempo se empezó a manifestar el Reynaud. Y en un principio me habían diagnosticado lupus, porque lo último, el último síntoma fue el endurecimiento de la piel, a mí me habían diagnosticado lupus y cuando ya la piel se puso durita pues ya me diagnosticaron. El reumatólogo me mandó una serie de pruebas o algo así. Lo que pasó es que me ingresaron durante 15 días porque yo estaba trabajando y me ingresaron pues para hacerlo mucho más rápido. Te hacen todas las pruebas seguidas y ya cuando salí de ahí ya salí con el diagnóstico de esclerodermia sistémica difusa. (Irma, 37 años. Esclerodermia)

El momento en que se recibe el diagnóstico se plantea como un punto de inflexión en la trayectoria del padecimiento y fue asumido de diversas maneras por nuestros entrevistados, en una gama de respuestas que van desde la ignorancia o negación, de la trascendencia de la información hasta el presagio de un porvenir bastante pesimista. Los adjetivos usados por el médico para calificar su condición tales como "crónica", "progresiva", "incurable", "irremediable", "degenerativa", "de causa desconocida" aparecen en la memoria de los entrevistados, como adjetivos cuyo significado no fue del todo comprendido en ese momento, sino con el paso del tiempo:

Una vez ya que fui al servicio de reumatología, con un simple análisis, el reumatólogo me diagnosticó que tenía una poliartritis, en aquel entonces la palabra que utilizó fue "una poliartritis crónica y progresiva". Así mismo me lo dijo. Yo no le di mucha importancia a las palabras "crónica" y "progresiva", no le di en aquel momento el significado tan importante como lo largo de mi vida al significado. Desconocía prácticamente una enfermedad crónica en una persona de 22 años y que fuera progresiva en aquel momento no me asustó, fue al cabo de unos días cuando uno se sitúa y se da cuenta de la magnitud de esas palabras. [El médico era] un hombre muy profesional y humano, duro pero humano, en el sentido de que no me engañó. Desde el primer día me dijo que yo tenía una artritis, que era crónica y que además eso iba ir a más. En aquel entonces lo de "progresiva" no lo había entendido, fue después y él me lo dijo [...] Fue un alivio saber por fin qué era lo que tenía. De primer momento la artritis decidió que iba a tener un desarrollo bastante importante, estaba afectando ambos pies y aquellos síntomas en la muñeca derecha $y$ algo en rodilla izquierda. (Jorge, 55 años. Artritis reumatoide)

En algunos casos, había una visión inmediatista de las consecuencias del diagnóstico, en la que lo que predominaba era la emergencia de los síntomas y el malestar que tenían, con la expectativa de que, a partir de identificar la entidad nosológica, la prescripción terapéutica sería eficaz y cesarían los síntomas para recobrar su salud, quitar el dolor, recuperar la movilidad, poder volver a realizar sus actividades normales. No se percibía claramente lo que podía significar a mediano o largo plazo:

Fue la primera vez que me dijeron que lo que tenía era Artritis reumatoide; por primera vez me lo dijeron. Una reumatóloga, me explicó el que mis rodillas, con el tiempo la solución última que tendría, que no habría más remedio, es la operación de ponerme prótesis en cada rodilla [...] eh, me dijo que esa operación tenía riesgo de quedarse la pierna rígida. Pero dijo, con el tiempo pues te lo tendrás que hacer. $Y$ entonces yo ahí no sabía nada de nada. Eso fue lo primero que me dijeron. Así fue mi diagnóstico. Creo que fue mejor, porque si de pronto me dicen todo lo que iba a tener que pasar hubiera sido peor: Me vengo abajo, me 
vengo abajo completamente. Así estuvo bien como me lo dijeron. [...] Cuando me explicaron la enfermedad, lo que me dijeron es que no, que no había cura. Me dijo que no había remedio. Que actualmente no había remedio o cura para esta enfermedad. Me explicaron que la artritis la ocasiona, de que las personas podrían morir diez años antes de la edad esperada. Yo estaba tan mal, que no me importó. No me importaron los años, es lo de menos que me importó. Lo que más me importaba a mí, era a ver si caminaba, y si caminaba bien, a ver si andaba y las escaleras no pasaba mucho porque normalmente tienes que subir o bajar pocas escaleras, pero más que nada, era el poder andar y si yo podía andar. Es que me dice gente que la esperanza de vida, por ejemplo que son 75 años. Y como yo era tan joven, tenía 21 años que me dijesen que de 65 años me iba a morir diez años antes, pues no me importaba, porque ahí ya caminaba como una yaya [abuela] digamos, como una viejita. En las escaleras era, yo digo como una yaya de 90 o 100 años. Lo que me preocupaba era si podría caminar, si podría caminar bien, si podría recuperar mi movilidad. (Jacinta, 38 años. Artritis juvenil).

Por otro lado, la manera en que el médico dice el diagnóstico y, sobre todo, el pronóstico que visualiza para el paciente, es fundamental para el impacto de la información. La contundencia de la noticia dependerá de la alarma o la relativa minimización del daño, con los respectivos impactos emocionales. Es relevante cómo nuestros informantes recuerdan con claridad la manera en que se les dijo el nombre de su padecer y el procesamiento que tuvieron que hacer en aquel momento.

Me la diagnostican [la espondilitis] de un modo muy bruto, pero quizá yo se los tengo que agradecer porque me hizo cambiar pues mucho. Me dijeron que era una enfermedad de la cual yo no me iba a morir, pero que la iba a tener que padecer toda la vida y que me cuidara mucho porque si no, me vería en una silla de ruedas. Me lo dijeron solo con 24 años y algo. Me hundió... [Felipe comienza a llorar] pero bueno, como yo tenía que seguir trabajando y tenía que seguir haciendo mi vida, pues seguí adelante. Se los tengo que agradecer porque, a lo mejor si me dicen que bueno no te preocupes, que no pasa nada, que tal... pues yo a lo mejor no sé, no me hubiera concienciado no tanto de que lo que yo tenía, era fuerte. Por eso es que esto se los tengo que agradecer...Te dan la noticia... Sí, pero, bueno yo que sé, a lo mejor ellos pensaron en que como verdaderamente era tan serio. Yo creo que pensaron que tenía que reaccionar, porque si no reaccionaba con lo que me dijeron, me podría quedar en la silla de ruedas... y de hecho no estoy porque la cirugía verdaderamente me ha ayudado mucho. Estoy operado de las dos caderas y gracias a eso no estoy en una silla de ruedas. (Felipe, 55 años. Espondilitis anquilosante)

Un elemento a destacar como constante es que a partir del diagnóstico ya es posible nombrar, conocer y actuar sobre la enfermedad, que hasta ese momento aparecía como una entidad desconocida o errática. Es relevante que, durante un largo tiempo, el sujeto no sabe a ciencia cierta cuál es la enfermedad crónica que lo aqueja, se emiten diagnósticos errados o difusos y se administran tratamientos que son simplemente paliativos de la sintomatología identificada. En este sentido, el diagnóstico también puede significar un alivio, una certeza después de un camino incierto y largo, que al menos les permite visualizar con mayor claridad el futuro. Se inicia otra etapa de la trayectoria de atención, en la que un largo proceso de duelo, con sus procesos de negación, huida hacia adelante, tristeza y resolución que se desarrollará con sus propios ritmos, crisis, periodos de estabilidad, adaptación, producto de su propia trayectoria del padecimiento: 
Un amigo me recomendó ir a ver al doctor G. al Hospital Clínico. [...] Es un médico muy especial, muy especial. Entonces él me estuvo convenciendo y mentalizando de lo que tenía que hacer y lo que no tenía que hacer. [...] Y bueno pues este hombre me introdujo en una especie de bienestar tremendo, por primera vez me dijeron lo que tenía. Me dijo que tenía una enfermedad que se Ilamaba Espondilo artritis, que era una enfermedad crónica que significaba que no habría en este momento curación, pero que llevando un tratamiento adecuado y haciendo la gimnasia pertinente que podía hacer una vida totalmente normal. Una vida laboral, familiar, sexual, normal. En ese momento yo Ilevaba 17 años con mi enfermedad. Asoció todo lo anterior, lo de las rodillas, del glúteo, la rigidez, el dolor, toda esa parte anterior la relacionó. [...] Si puedo decirte las sensaciones [...] salí con la sensación, con el convencimiento de que tenía una enfermedad incurable. Una conciencia de tener una enfermedad para toda la vida, pero con la seguridad de que podía hacer una vida normal y sana, esa es la sensación que tenía. La sensación de alivio de... bueno casi, casi de felicidad, por encontrar alguien que cuida de $t i$, que se preocupa, que te quiere ayudar. Claro, no me cobró. Ni esa visita, ni las posteriores durante seis o siete años. Es muy especial, sí, sí., es como un loco, una persona que está fuera de los parámetros sociales y [...] bueno, a ver, no con todo el mundo hace eso, pero con mucha gente, la que él considera que está muy necesitada lo ha hecho. (Pedro, 60 años. Espondilitis y ceguera)

La información que provee el médico puede resultar, hasta cierto punto, suficiente en cuanto a lo que el paciente puede procesar en ese momento. Sin embargo, con posterioridad comenzarán a surgir más preguntas y nuevas incertidumbres en la configuración del futuro. La perspectiva de atender y solucionar los síntomas, de retorno a la vida cotidiana, es la expectativa inicial que, al cabo de un tiempo, se reconoce como un punto de no retorno, como un trastorno en la biografía del sujeto que, sin embargo, se vincula con una historia previa.

El saber médico reconoce sus limitaciones terapéuticas y las lagunas en sus propios modelos explicativos de la enfermedad y, de alguna manera, coloca al sujeto delante de la imposibilidad de la cura, los tratamientos que tendrán una eficacia paliativa del dolor, la significación de la cronicidad como nueva forma de vivenciar el padecimiento y acostumbrarse a sufrirlo, con aceptación y estoicismo, con el aparente consuelo de que esa no será la causa de muerte.

En este sentido, conviene señalar la importancia de la relación médico paciente en cada una de las narrativas. En todos los casos, se hace una evaluación del saber técnico del médico pero, sobre todo, de la calidad humana, de la sensibilidad o dureza con que comunica la noticia, el tiempo destinado a explicar la condición médica del paciente, el posible pronóstico y la indicación terapéutica.

\section{CONCLUSIONES}

Desde la mirada retrospectiva de las personas entrevistadas, el diagnóstico definitivo representa la respuesta central a la pregunta ¿qué me está pasando?, cuestión que, en el caso de las enfermedades reumáticas, puede constituirse en un itinerario hasta cierto punto difuso o errático, terapéuticas paliativas de determinados síntomas, que en la memoria de nuestros informantes es recreada como un periodo en el que la incertidumbre se suma a la ansiedad y el malestar derivado del padecimiento.

A la luz de los años transcurridos desde aquel momento, el diagnóstico definitivo se identifica como el punto inicial de una larga trayectoria, de ahí la pertinencia de aplicar el concepto de disrupción biográfica para explicar la alteración en el rumbo, y la dificultad con el curso normal de la vida hasta 
ese momento. Al reconstruir la trayectoria del padecimiento se pueden identificar otros puntos de inflexión como las cirugías de colocación de prótesis, las infiltraciones, los ingresos hospitalarios, entre otras. Asimismo, se hallan otros puntos de no retorno, tales como el retiro de la vida laboral, la obtención de la pensión por enfermedad y discapacidad; el desprendimiento o disolución de los vínculos sociales, dejar de realizar ciertas actividades sociales o lúdicas que, como consecuencia de la enfermedad, se van perdiendo a través del tiempo.

Tomando como eje la trayectoria del padecimiento pudimos establecer la etapa correspondiente al itinerario diagnóstico y las múltiples significaciones que el momento del diagnóstico tuvo en la biografía del sujeto. En cada una de las narrativas mostradas, es posible aproximarnos a las sensaciones, emociones, aflicciones y aprendizajes reconocidos como producto de esta experiencia diagnóstica, que han llevado a los sujetos por un laberinto de incertidumbre, dolor y búsqueda de respuestas y soluciones. El diagnóstico, como momento disruptivo, permite reinterpretar el pasado del padecimiento -un periodo en el que el padecer no tiene nombre o es equívoco- y enfocarse hacia el futuro, ahora con una forma de reconocerlo, nombrarlo, un encuadre nosológico y terapéutico en el que puede posicionarse y generar estrategias de afrontamiento, también significará una etiqueta social que tendrá repercusiones en su propia identidad, en la percepción de sí mismo y la que los otros tienen de él o ella, la autoimagen, la imagen que proyecta y la que se le devuelve en la mirada de los otros, en las relaciones sociales que se activan y que se desactivan y en la estructura social y cultural que lo constriñe a optar por ciertas estrategias y descartar otras, que moldea su nuevo estilo de vida.

En este trabajo se ha abordado tan solo una etapa de toda esta trayectoria que da cuenta del impacto y significación del diagnóstico como un momento de disrupción en la biografía del sujeto, a partir del cual encontrará nuevos cauces para afrontar su condición.

Considero necesario abrir los espacios para que las voces de los afectados -los enfermos y sus familias- sean escuchadas, propiciando una mayor sensibilización de la población y, en última instancia, buscar alternativas que mejoren la calidad de vida de las personas afectadas, y ayuden a vivir a través de la cronicidad.

\section{REFERENCIAS BIBLIOGRÁFICAS}

1. Osorio RM. La experiencia de los padecimientos reumáticos en un grupo de autoayuda en la ciudad de Barcelona: desafíos y dilemas en la investigación sobre enfermedades crónicas. En: Romaní O, Larrea C, Fernández, J, (coord.). Antropología de la medicina, metodologías e interdisciplinariedad: de las teorías a las prácticas académicas y profesionales. Donostia: ANKULEGI Antropologia Elkartea; 2008. p. 157-172.

2. Csordas T, (ed.). Embodiment and experience: The existential ground of culture and self. Cambridge: Cambridge University Press; 1994.
3. Bourdieu P. La ilusión biográfica. En: Bourdieu P. Razones prácticas sobre la teoría de la acción. Barcelona: Anagrama; 1997.

4. Young JT. Illness behaviour: a selective review and synthesis. Sociology of Health and Illness. 2004;26(1):1-31.

5. Murphy RF. The damage self. En: Brown PJ, (ed.). Understanding and applying medical anthropology. Mountain View: Mayfield Publishing; 1998.

6. Zola IK. Self, identity and the naming question: Reflections on the language of disability. In: Henderson GH, King NMP, Strauss RP, Estroff 
S, Churchill L, (ed.). The social medicine reader. Durham-London: Duke University Press; 1997.

7. Bury M. Chronic illness as biographical disruption. Sociology of Health and Illness. 1982;4(2):167-182.

8. Boeijea GM, Hennie $R$, Duijnstee $M S H$, Grypdonckb MHF, Poolc A. Encountering the downward phase: biographical work in people with multiple sclerosis living at home. Social Science \& Medicine. 2002;55:881-893.

9. Bury M, Gabe J, (ed.). The sociology of health and illness: A reader. London: Routledge Student Readers; 2004.

10. Gregory S. Living with chronic illness in the family setting. Sociology of Health \& Illness. 2005;27(3):372-392.

11. Becker G. Coping with stigma: Lifelong adaptation of deaf people. In: Brown PJ, (ed.). Understanding and applying medical anthropology. Mountain View: Mayfield Publishing; 1998.

12. Goffman E. Estigma: La identidad deteriorada. Buenos Aires: Amorrortu; 1970.

13. Williams SJ. Is anybody there? Critical realism chronic illness and the disability debate. Sociology of Health and IIIness.1999;21(6):797-819.

14. Williams SJ. Chronic illness as biographical disruption or biographical disruption as chronic illness? Reflections on a core concept. Sociology of Health and IIIness. 2000;22(1):40-67.

15. Williams SJ. Medicine and the body. London: Sage Publications; 2003.

16. Carricaburu D, Pierret J. From biographical disruption to biographical reinforcement: the case of HIV-positive men. Sociology of Health and Illness. 1995;17(1):65.

17. Adamson J, Donovan J. Normal disruption: South Asian and African/Caribbean relatives caring for an older family member in UK. Social Science \& Medicine. 2005;60:37-48.

18. Faircloth CA, Boylstein C, Rittman M, Young $\mathrm{ML}$, Gubrium J, Sudden illness and biographical flow in narratives of stroke recovery. Sociology of Health \& IIIness 2004;26(2):242-261.

19. Charmaz K. Loss of self: a fundamental form of suffering in the chronically ill. Sociology of Health and Illness. 1983;5(2):168-195.

20. Charmaz K. Good days, bad days: The self in chronic ilness and time. New Jersey: Rutgers University Press; 1997.

21. Manderson L, Smith-Morris C, (ed.). Chronic conditions, fluid states: Chronicity and the anthropology of illness. New Brunswick: Rutgers University Press; 2010.

22. Kleinman A. The illness narratives: Suffering, healing and the human condition. New York: Basic Books; 1988.

23. Strauss A, Corbin J, Fagerhaugh S, Maines D, Glaser B, Sucsek B, Wiener C. Chronic illness and the quality of life. St Louis: C.V. Mosby Company; 1984.

24. Corbin J, Strauss AL. Unending work and care. San Francisco: Jossey Bass Publishers; 1988.

25. Burish TG, Bradley LA, (ed.). Coping with chronic disease: Research and applications. New York: Academic Press; 1983. 